\title{
High Resolution Electron Microscopy Analysis of the Helicobacter pylori Cag Type IV Secretion System
}

\author{
Jennifer A. Gaddy ${ }^{1}$ and Timothy L. Cover ${ }^{1,2}$ \\ 1. Vanderbilt University School of Medicine, Nashville, TN, USA \\ 2. Veterans Affairs Tennessee Valley Healthcare System, Nashville, TN, USA
}

Helicobacter pylori is a gram negative bacterium that colonizes half of the world's population. Infection with this organism is a strong risk factor for peptic ulcer disease and gastric adenocarcinoma. H. pylori strains containing the cag pathogenicity island (PAI) are associated with a higher incidence of disease, compared to strains that lack the cag PAI. The cag pathogenicity island encodes components of a type IV secretion system (T4SS) that is required for translocation of the oncogenic effector molecule, CagA, into host epithelial cells, leading to changes in cell signal cascades that potentially lead to carcinogenesis. Production of pilus-like structures by $H$. pylori has been linked to the cag PAI and cag T4SS-dependent phenotypes (1). The goals of the current study were to identify $H$. pylori genes involved in biogenesis of cag T4SS-associated pili and to identify environmental signals that regulate the assembly of these structures.

High resolution scanning electron microscopy (SEM) analysis revealed the formation of pilus-like structures (measuring about $13 \mathrm{~nm}$ in width and $65 \mathrm{~nm}$ in length) at the bacteria-host cell interface when $H$. pylori was co-cultured with AGS gastric epithelial cells. In contrast, these structures were rare or absent when bacteria were cultured in the absence of gastric epithelial cells. By analyzing a panel of $H$. pylori mutant strains and complemented mutants, we have identified at least four genes in the cag PAI (cagE, cagI, cagL, and cagT) that are required for pilus formation. A $\triangle c a g H$ mutant strain produces pili that are thicker, longer and more abundant than those produced by the wild-type strain, which suggests that $\mathrm{cagH}$ is a regulator of pilus dimensions. Immuno-electron analysis revealed that $\mathrm{CagH}, \mathrm{CagI}$ and $\mathrm{CagL}$ localize to the surface of the bacterial cell (3). A $\Delta c a g Y$ mutant strain produces pili, but the surface of these mutant bacteria and the tips of the T4SS pili are decorated with reduced amounts of CagA compared to what is observed with the wild-type strain, based on immunogold-EM analysis. Therefore, CagY has a role in CagA export to the bacterial cell surface (4).

Co-culture of $H$. pylori with AGS cells under conditions of low iron availability resulted in a significant increase in pilus production and increased T4SS activity. The addition of an exogenous iron source resulted in inhibition of pilus production and reduced T4SS activity (2). qRT-PCR demonstrated that the transcript abundance of several cag genes is increased under conditions of low iron availability.

Taken together, these results indicate that several genes in the cag PAI are required for pilus biogenesis. The $\operatorname{cagH}$ gene is involved in the regulation of pilus dimensions. The $\operatorname{cag} Y$ gene is important in CagA decoration of the bacterial outer membrane, but is dispensable for pilus biogenesis. Enhanced pilus formation in response to low-iron conditions is mediated at least in part by increased transcription of genes encoding T4SS components (5). The observed effects of low iron conditions on T4SS-related phenotypes may help to explain the increased risk of gastric cancer that occurs in individuals with iron-deficiency anemia.

\section{References:}

[1] Johnson, E.M., Gaddy, J.A., and Cover, T.L. Front. Cell. Infect. Microbiol. 2:17 (2012), 10.3398.

[2] Noto, J.M et al. J Clin Invest 123:1(2013), 479-92.

[3] Shaffer, C.L. et al PLoS Pathogen 7:9 (2011), e1002237.

[4] Barrozo, R.M. et al PLoS Pathogen (2013) in press.

[5] The authors acknowledge funding P01CA116087, R01 AI068009, AI039657 and Dept. of Veterans

Affairs (to T.L. Cover) and F32 AI102568 and Dept. of Veterans Affairs (to J.A. Gaddy). 
Microsc. Microanal. 19 (Suppl 2), 2013 